\section{Relationship of a Novel c.429delC Deletion in Hairless Gene HR with Alopecia in Two Families from Southern Punjab, Pakistan}

\author{
Muhammad Ajmal', Saima Mustafa ${ }^{1}$, Fizza Ibrahim Bajwa ${ }^{1}$, Cheng Zhou', \\ Guangdong Wen², Soe Lwin Myint ${ }^{2}$, Syed Irfan Raza ${ }^{3}$, Ihtasham Bukhari, \\ Mubashir Hassan ${ }^{5}$, Muhammad Faisal ${ }^{6}$ and Furhan Iqbal ${ }^{1 *}$ \\ ${ }^{1}$ Institute of Pure and Applied Biology, Zoology Division. Bahauddin Zakariya \\ University Multan 60800, Pakistan. \\ ${ }^{2}$ Department of Dermatology, Peking University People's Hospital No.11 Xizhimen \\ South Street, 100044 Beijing, People Republic of China. \\ ${ }^{3}$ Department of Biochemistry, HBS Medical College, Shaheed Zulfiqar Alu Bhutto \\ Medical University, Islamabad, Pakistan. \\ ${ }^{4}$ Translational Research institute, Henan Provincial People's Hospital, School of \\ Medicine, Henan University, Huanghe Road, Zhengzhou, Henan 450053, China. \\ ${ }^{5}$ Institute of Molecular Biology and Biotechnology/(IMBB), The University of Lahore, \\ Pakistan \\ ${ }^{6}$ Faculty of Health Studies, University of Bradford, United Kingdom
}

Muhammad Ajmal and Saima Mustafa have contributed equally to the manuscript.

\section{A B S T R A C T}

Atrichia with papular lesions (APL) is a rare autosomal recessive form of total alopecia, characterized by hair loss soon after birth and the development of papular lesions of keratin-filled cysts over extensive areas of the body. Two consanguineous families were enrolled from Basti Mochi Wala, Mouza Gulab Shah in Muzaffargarh District of Punjab (Pakistan) having multiple siblings suffering from alopecia. The aim of this study was to find out the genetic mutation(s) in hairless $(H R)$ gene, if any, in the enrolled subjects. A questionnaire was filled for each subject on the sampling site in order to collect epidemiological data associated with the disease. Patients from both families exhibited congenital atrichia with papular lesions (APL) including hair loss in the scalp, pubic and other body parts. Polymerase chain reaction (PCR) was used to amplify all the over lapping intron exon regions of $H R$ gene followed by DNA sequencing. Analysis of the DNA sequence revealed a novel deletion c.429delC in exon 2 of HR gene. Due to this deletion Proline at 144 changed into Lysine causing frame shift leading to premature termiation of the protein after 23 amino acid residues (p.P144LfsX23), resulting in a truncated HR protein with 166 amino acid residues. The mutation followed Mendalian pattern of inheritance as all the patients are homozygous for the mutation while parents were heterozygous and unaffected siblings from both families were either heterozygous for the reported mutations or they lacked this mutation.

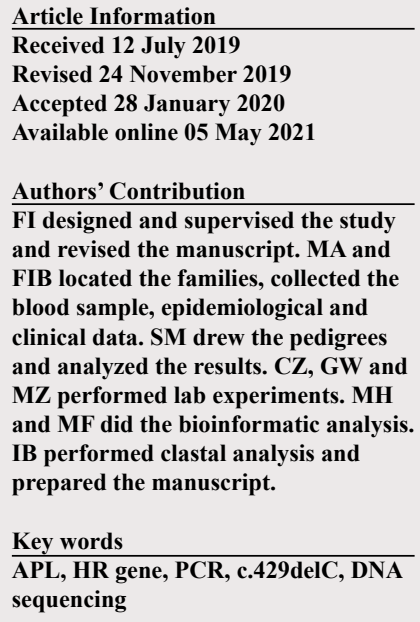

Key words

APL, HR gene, PCR, c.429delC, DNA sequencing

\section{INTRODUCTION}

A lopecia is a problem affecting primates worldwide with an approximate incidence of $68 \%$. Its exact etiology is not known but some causes include aging, nutritional deficiencies, endocrine disorders and stress (Kramer et al., 2010; Ahmed et al., 2013). It is also caused due to inflammatory diseases, anemia and infections (Macias et al., 2013). Alopecia has seven major sub-types

\footnotetext{
* Corresponding author: furhan.iqbal@bzu.edu.pk 0030-9923/2021/0004-1243 \$ 9.00/0

Copyright 2021 Zoological Society of Pakistan
}

depending upon the intensity and pattern of hair loss (Hammerschmidt and Brenner, 2014): alopecia areata (AA), alopecia totalis (AT), alopecia universalis (AU), androgenetic alopecia (AGA) and telogen Effluvium (TE). In addition to these, chemotherapy induced alopecia (CIA), cicatricial and non-cicatricial alopecia have also been reported in literature (Werner and Brenner, 2012; Amin and Sachdeva, 2013; Filbrandt et al., 2013; Santos et al., 2015).

The $H R$ gene has been identified and mapped on human chromosome $8 \mathrm{p} 12$ and spans genetic distance of $14 \mathrm{~kb}$ and consist of 19 exons (Novak and Meyer, 2009). Exon 17 has 2 isoforms produced due to the alternative 
usage of hairless gene in human tissues (Werner and Brenner, 2012). The $H R$ gene product is a transcriptional co-regulating factor having single zinc finger domain, which is expressed highly in brain and skin (Kraemer et al., 2008; Kramer et al., 2010). HR protein has been shown to interact with multiple nuclear receptors, including thyroid hormone receptor (TR), the retinoic acid receptor-related orphan receptors (ROR) and the vitamin D receptors (VDR) (Hsieh et al., 2003). Up till now, more than 38 mutations have been reported in $H R$ gene (MIM 602302) including deletions, non-sense, missense, insertions, and splice site and compound mutations (Kraemer et al., 2008). The aim of this study was to determine the molecular basis of alopecia in two consanguineous families from Southern Punjab by screening the $H R$ gene in all enrolled subjects and to compare the genotype and phenotypic associations in them.

\section{MATERIALS AND METHODS}

\section{Blood and data collection}

In the present study, two consanguineous families were enrolled from Basti Mochi Wala, Mouza Gulab Shah in Muzaffargarh District in Punjab (Pakistan) having multiple siblings suffering from alopecia. Six blood samples were collected from family one (PK-ALO-01) including two patients and four controls (Fig. 1A). While nine blood samples were collected from second family (PK-ALO-02) including three patients and six controls (Fig. 1B). Blood was sampled from median cubital vein and preserved in $1.5 \mathrm{ml}$ Eppendorf tube containing 0.5M EDTA solution after having written informed consent from the enrolled subjects. A questionnaire was filled in for each subject on the sampling site in order to collect epidemiological data associated with alopecia, if any. All the experimental protocols and subject handling procedures were approved by the ethical committee of Institute of Pure and Applied Biology, Bahauddin Zakariya University Multan, Pakistan.

\section{DNA extraction}

Inorganic method was used for DNA extraction targeting the white blood cells following Taqddus et al. (2014).

\section{Genotyping in HR gene}

List of primers used to amplify the 19 exons of $H R$ gene along with overlapping intronic regions are mentioned in Supplementary Table I. Polymerase chain reaction was carried out in a total volume of $50 \mu \mathrm{l}$. PCR reaction mixture consisted $2 \mu$ l deoxynucleotide triphosphate (dNTPs), $2 \mu \mathrm{l}$ of each primer, $2.5 \mu \mathrm{l}$ of buffer $\mathrm{A}, 1 \mu \mathrm{l}$ of divalent cation $\mathrm{MgCl}_{2}, 5 \mu \mathrm{l}$ of DNA template, $0.7 \mu 1$ of replication starting enzyme, Taq DNA polymerase (Vivantis, UK). Amplification of DNA was processed in a DNA thermocycler (MultiGene OptiMax PCR system 1402015). Thermal profile conditions were $94^{\circ} \mathrm{C}$ for $5 \mathrm{~min}$, followed by 35 cycles of $94^{\circ} \mathrm{C}$ for 30 seconds, annealing temperature for 30 seconds, $72^{\circ} \mathrm{C}$ for 45 seconds, and then final extension at $72^{\circ} \mathrm{C}$ for $10 \mathrm{~min}$. The annealing temperature of the reaction is decreased $1^{\circ} \mathrm{C}$ every cycle from $62^{\circ} \mathrm{C}$ to a 'touchdown' at $55^{\circ} \mathrm{C}$, at which temperature, 27 cycles are carried out. Amplified DNA products were separated according to their molecular weight by electrophoresis through $2 \%$ agarose gel.

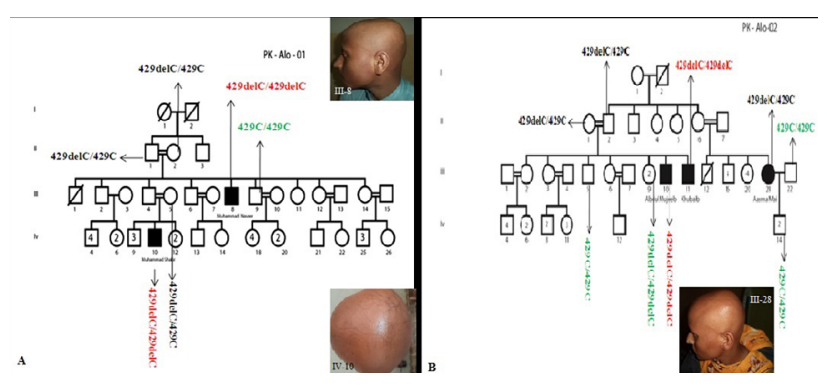

Fig. 1. Pedigree and genotyping results for families PKALO-01 and 02 at nucleotide 429 (exon 2) of $H R$ gene along with phenotypic appearance of affected subjects in family PK-ALO-01; Subject III: 8 had no eye-lashes and scalp hairs, Subject IV: 10 had no eye-lashes with paucity of scalp hairs. In PK-ALO-02, subject III: 28 had no eyelashes and scalp hairs.

\section{DNA ssequencing and analysis}

The $H R$ gene was amplified by specially designed oligonucleotide primers (Supplementary Table I) and the PCR products were purified by shrimp alkaline phosphatase and exonuclease enzymes and sequenced by using 3130 Genetic Analyzer (Applied Biosystems, Foster City, CA). The sequencing files were inspected by using SeqMan software (DNAstar Lasergene version 5.0.221.0) following Bukhari et al. (2017).

\section{Multiple ssequence an alignment analysis}

Sequences of HR protein for different vertebrates were taken from NCBI protein https://www.ncbi.nlm.nih. gov/protein/ and uploaded in to Clastal Omega online tool https:/www.ebi.ac.uk/Tools/msa/clustalo/ for multiple sequence alignment and evolutional conservation analysis.

\section{Prediction of $3 D$ structure of HR protein}

The three dimensional (3D) crystal structures of HR protein were not reported in Protein Data Bank database (http://www.rcsb.org). Therefore, a homology modeling approach was employed to predict 3D structure of HR. 
The amino acid sequences in FASTA format of HR protein having Uniprot ID O43593 was retrieved from UniProt Knowledge database (https://www.uniprot.org/uniprot/ O43593). For building of protein models selection of the correct template is most significant parameter to predict the good protein model (Sadowski and Jones, 2007). The human template structure with PDBID \# 5FZO was selected for prediction of HR protein structure on the basis of sequence identity (33\%). Moreover, for building of mutant protein model PDBID 4II4 was employed with sequence identity $(31.43 \%)$. Mutation in protein results in de-stability in protein structure which may results in abnormalities in human like melanogenesis (Hassan et al., 2018). The three dimensional (3D) crystal structures of HR protein was not reported in Protein Data Bank database (http://www.rcsb.org). Therefore, a homology modeling approach was employed to predict 3D structure of HR. The amino acid sequences in FASTA format of HR protein having Uniprot ID 043593 was retrieved from UniProt Knowledge database (https://www.uniprot.org/uniprot/ O43593). For building of protein models selection of the correct template is most significant parameter to predict the good protein model (Sadowski and Jones, 2007).

\section{RESULTS}

\section{Clinical findings}

Subjects enrolled from family PK-ALO-01 included two alopecia patients. Subject III:8 was born with scalp hairs and lost his hairs gradually and around the age of three years lost all his scalp hairs. Eye-lashes were also absent. Whereas the patient IV:10 was born without scalp hairs and eye-lashes. Subjects enrolled from family PKALO-02 included three patients. Subject III:10 was born with normal scalp hairs and lost his hairs gradually and around the age of seven years, he lost all his scalp hairs and eye-lashes were also absent. Whereas the patient III: 11 was born with normal scalp hairs and lost his hairs gradually till he was seven years. Eye-lashes are absent but paucity of scalp hairs was still visible at sampling time. The third patient, III:21 was born with normal scalp hairs and lost her hairs gradually and around the age of seven years, she lost all her scalp hairs and eye-lashes were also absent (Fig. 1). While all other members of both families had normal scalp, body hairs and eye-lashes. The epidemiological data of both families has been documented in Table I.

\section{Mutation screening in HR gene}

DNA sequence analysis revealed that both alopecia patients in family PK-ALO-01 and all three patients in family PK-ALO-02 were homozygous for a novel deletion c.429delC in exon 2 of $H R$ gene (Fig. 1A, B) while the normal siblings of both families were either heterozygous for above mentioned mutation or they had normal ' $\mathrm{C}$ ' nucleotide at position 429 in exon 2 (Fig. 2). Due to this deletion Proline at 144 changed into Lysin causing framshif leading to premature termiation of the protein after 23 amino acid residues (p.P144LfsX23), resulting in a truncated HR protein with 166 amino acid residues.

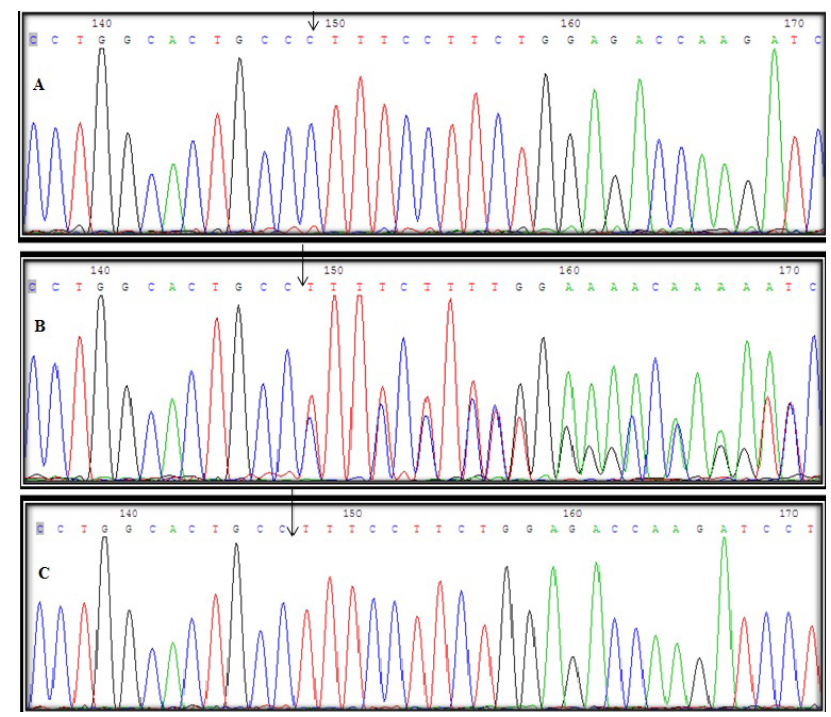

Fig. 2. Histogram showing the DNA sequence analysis of $H R$ gene mutation. A, Wild type sequence at position 429 in subject III:9 (PK-ALO-01); B, Heterozygous sequence in subject II:1 (PK-ALO-01); C, Homozygous deletion of a nucleotide $\mathrm{C}$ at position 429 in subject III: 10 (PK-ALO-02).

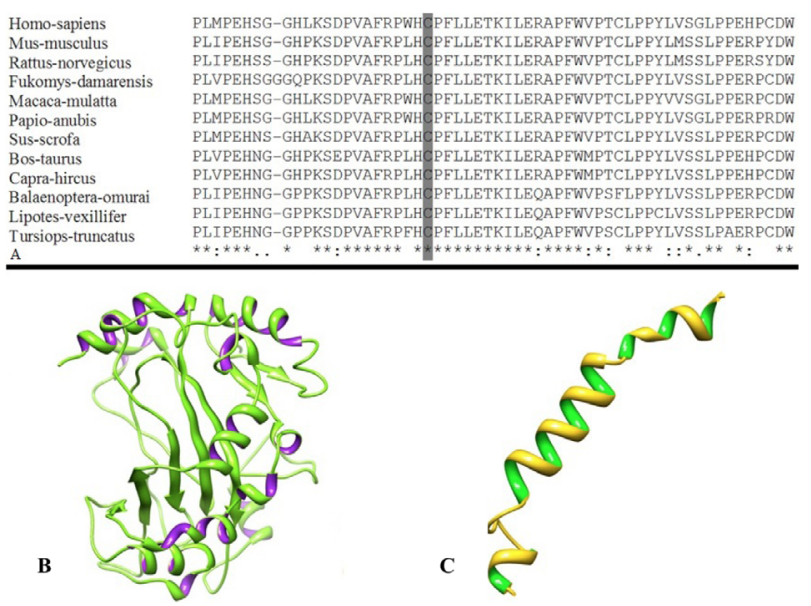

Fig. 3. A, Clastal omega analysis of HR protein in different vertebrates. Mutant amino acid Cysteine is highly conserved among all species and highlighted gray. Asterisks represent the highly conserved amino acids; $\mathbf{B}$, The predicted structure of wild protein; $\mathbf{C}$, mutant protein structure. 


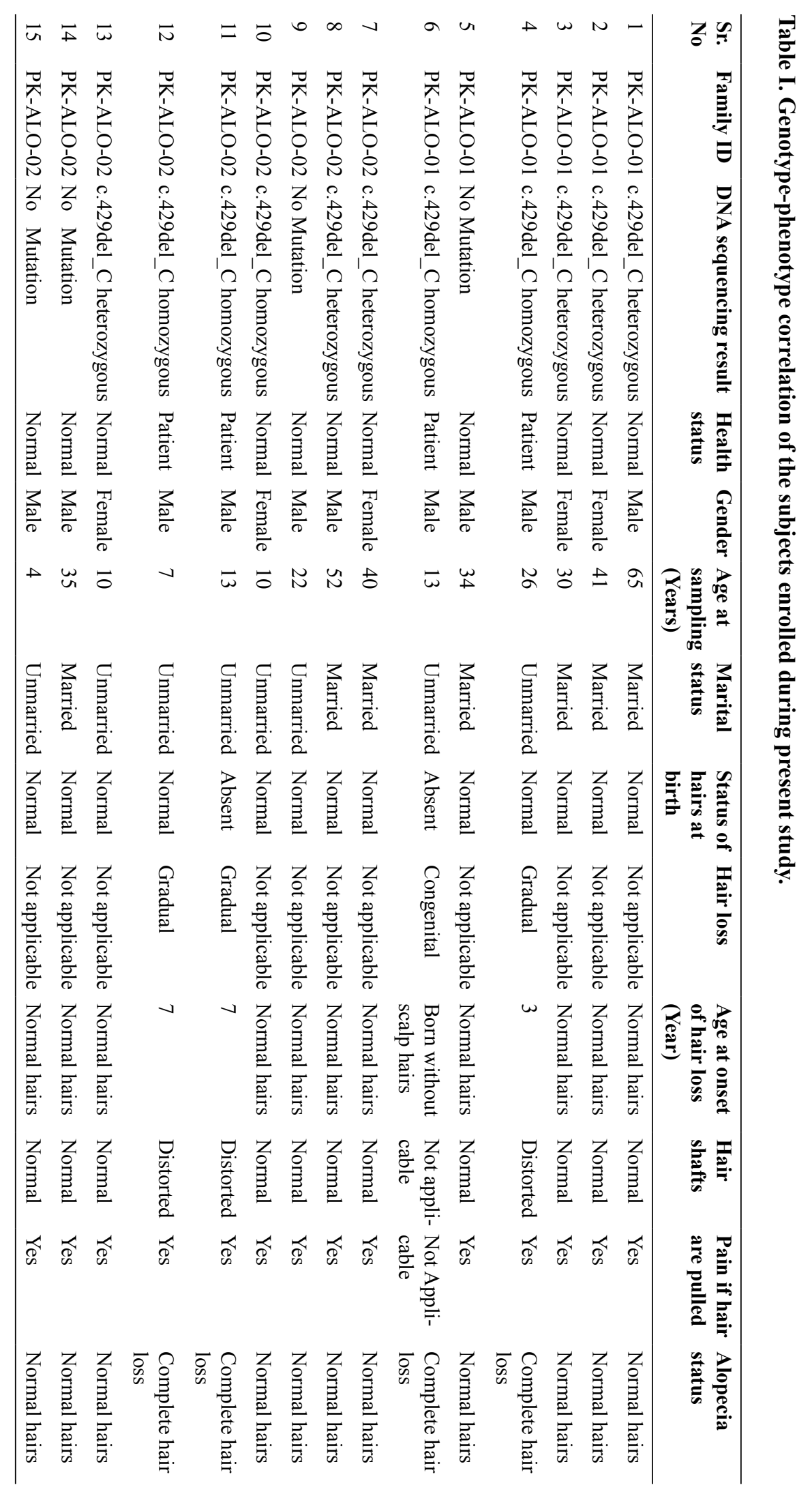


Clustal Omega analysis revealed that Cysteine and other amino acids of this domain are highly conserved in vertebrates (Fig. 3A) and any mutation in this region may have profound effects on the phenotype of the individuals.

\section{Prediction of $3 D$ structure of HR protein}

The human template structure with PDBID \# 5FZO was selected for prediction of HR protein structure on the basis of sequence identity (33\%). Moreover, for building of mutant protein model PDBID 4II4 was employed with sequence identity $(31.43 \%)$. Mutation in protein structure resulted in destability in its structure which may results in abnormalities in human like melanogenesis (Fig. 3B, C) (Hassan et al., 2017).

\section{DISCUSSION}

Congenital atrichia (CA) is the total absence of body hair from birth. It is an inherited condition and is associated with papular lesions on the neck, face, trunk or limbs that are not present at birth but develop later in life (Kirit and Parthasaradhi, 2014). It is characterized by the deformity of hair follicles causing early onset of hair loss (Pedrosa et al., 2013). It is usually associated with a mutation in a $H R$ gene located at chromosome 8 that can be inherited as autosomal recessive or autosomal dominant (Kirit and Parthasaradhi, 2014). Sometimes, at birth scalp hairs can be present that are shed later after few months and hair re-growth does not occur. It is considered as total alopecia of eye-lashes, eye-brows and general body hair (Sacchidanand et al., 2012). The objective of this study was to determine the molecular basis of alopecia in two consanguineous families from Southern Punjab.

The $H R$ gene appears to function as nuclear receptor co-repressor (Sacchidanand et al., 2012). The nuclear receptors are actually transcriptional factors and their activity depends upon activity of additional proteins. These additional proteins are known as co-repressor are found to be involved in chromatin remodeling (Jespen and Rosenfeld, 2002). The functional role of $H R$ gene has been confirmed in HR mutant mice. Initial hair growth and hair follicle morphogenesis was normal in hr mutant mice. It was observed that when hair follicles relapsed, telogen stage does not re-enter into anagen stage and the hair shed off and new hairs were not then produced causing alopecia (Panteleyev et al., 1999).

In present study, two consanguineous families (ALO01 and ALO-02) demonstrating alopecia were ascertained from Southern region of Punjab. The mode of inheritance was autosomal recessive in both families. In both families, affected individuals demonstrated congenital atrichia. In these two families, sequence analysis of $H R$ gene revealed novel deletion mutation (c.429delC) causing frameshift and a premature termination (p.P144LfsX23) of HR. Premature termination codon (PTC) in an mRNA open reading frame causes inactivation of gene function by production of truncated protein and hence destabilization of mRNA (John et al., 2005). Mutations in HR gene have previously been reported in consanguineous families from Pakistan. John et al. (2005) had reported for the first time a mutation in $H R$ gene that lead to frame shift and generation of pre-mature termination codon in two Pakistani families. Patients of both families exhibited congenital atrichia with APL including hair loss in the scalp, pubic and other body parts. Affected individuals of family A had keratin filled follicular cysts on thighs while affected individuals of family B had these follicular cysts on face and scalp. In Family A, single base pair deletion mutation at position 431 (431 delC) was observed while in Family B, single base pair deletion of $\mathrm{G}$ at nucleotide position 2021 (2021delG) was reported (Werner and Brenner, 2012).

In another study, Wali et al. (2006) reported that mutation in $H R$ gene caused the pathogenesis of atrichia with papular lesions (APL) in a Pakistani consanguineous family. The papular lesions of keratin-filled cysts over an extensive area of the body have been observed in the affected individuals of the family. Sequence analysis revealed an insertion of $11 \mathrm{bp}$ repeated sequence at nucleotide position 202 [202(Ins CTTCCCCCAGG)] leading to frame shift and pre-mature termination codon (Wali et al., 2006).

In a recent study by Ahmed et al. (2013), a Pakistani family from Kyber Pukhtunkhwa province was reported comprising two individuals with clinical features of congenital atrichia with papular lesions. DNA sequencing revealed a novel missense mutation in $H R$ gene (G-to-A substitution at amino acid position 285) resulting in substitution of hydrophobic glycine (AGG) to polar serine (AAG) residue causing complete loss of body hairs but affected individuals are physically and mentally fit (Ahmed et al., 2013).

\section{CONCLUSION}

In conclusion, we have reported a novel deletion (c.429delC) in exon 2 of $H R$ gene in two consanguineous families. Review of literature has revealed a series of deletions and insertion mutations in $H R$ gene from Pakistani families suffering from alopecia confirming that mutations in this gene can lead to a variety of hair loss conditions in local population. 


\section{ACKNOWLEDGEMENT}

This research project was partly supported by grants from the National Natural Science Foundation of China (No. 81201221).

\section{Supplementary material}

There is supplementary material associated with this article. Access the material online at: https://dx.doi. org/10.17582/journal.pjz/20190712080701

\section{Conflict of interest statement}

Authors have declared no conflict of interest.

\section{REFERENCE}

Ahmad, W., Zlotogorski, A., Panteleyev, A.A., Lam, H., Ahmad, M., Ul Haque, M.F., Abdalah, H.M., Dragan, L. and Christiano, A.M., 1999. Genomic organization of the human hairless gene $(H R)$ and identification of a mutation underlying congenital atrichia in an Arab Palestinian family. J. Genom., 56: 141-148. https://doi.org/10.1006/geno.1998.5699

Ahmed, M.S., Rauf, S., Naeem, M., Khan, M.N. and Mir, A., 2013. Identification of novel mutation in the $H R$ gene responsible for atrichia with papular lesions in a Pakistani family. J. Dermatol., 40: $927-$ 947. https://doi.org/10.1111/1346-8138.12266

Amin, S.S., and Sachdeva, S., 2013. Alopecia areata: A review. J. Dermatol. Dermatol. Surg., 17: 37-45. https://doi.org/10.1016/j.jssdds.2013.05.004

Bukhari, I., Li, G., Wang, L., Iqbal, F., Zhang, H. and Yuanwei, Z., 2017. Effects of androgen receptormutation on testicular histopathology of patient having complete androgen insensitivity. $J$. mol. Histol., 48: 159-167. https://doi.org/10.1007/ s10735-017-9714-7

Cichon, S., Kruse, R., Hillmer, A.M., Kukuk, G., Anker, M., Altland, K., Knapp, M., Propping, P. and Nothen, M.M., 2000, A distinct gene close to the hairless locus on chromosome 8p21 underlies hereditary Marie Unna type hypotrichosis in a German family. Br. J. Dermatol., 143: 811-814. https://doi.org/10.1046/j.1365-2133.2000.03781.x

Filbrandt, R., Rufaut, N., Jones, L. and Sinclair, R., 2013. Primary cicatricial alopecia_diagnosis and treatment primary cicatricial alopecia: diagnosis and treatment. Can. med. Assoc. J., 185: 15791585. https://doi.org/10.1503/cmaj.111570

Hammerschmidt, M. and Brenner, F.M. 2014. Efficacy and safety of methotrexate in alopecia areata. $J$. Anais Brasil. Dermatol., 89: 729-734. https://doi. org/10.1590/abd1806-4841.20142869

Hassan, M., Abbas, Q., Raza, H., Moustafa, A.A. and Seo, S.Y., 2017. Computational analysis of histidine mutations on the structural stability of human tyrosinases leading to albinism insurgence. Mol. Biol. Sys., 13: 1534-1544. https://doi.org/10.1039/ C7MB00211D

Hsieh, J.C., Sisks, J.C., Jurutka, P.W., Hausslert, C.A., Slater, A.S., Hausslert, M.R. and Thompson, C.C., 2003. Physical and functional interaction between the vitamin D receptor and hairless corepressor, two proteins required for hair cycling. J. biol. Chem., 278: 38665-38674. https://doi.org/10.1074/ jbc.M304886200

Jespen, K. and Rosenfeld, M.G., 2002. Biological roles and mechanistic actions of co-repressor complexes. J. Cell Sci., 15: 689-98.

John, P., Aslam, M., Rafiq, M.A., Amin-ud-din, M., Haque, S. and Ahmad, W., 2005. Atrichia with papular lesions in two Pakistani consanguineous families resulting from mutations in the human hairless gene. J. Arch. Dermatol. Res., 297: 226230. https://doi.org/10.1007/s00403-005-0593-5

Kirit, R.E.P. and Parthasaradhi, A., 2014. Congenital atrichia associated with nevus flemmeus: A rare association. Ind. Dermatol. Online J., 5: 475-477. https://doi.org/10.4103/2229-5178.142505

Kraemer, L., Wajid, M., Shimomura, Y. and Christiano, A.M., 2008. Mutations in the hairless gene underlies APL in three families of Pakistani origin. $J$. Dermatol. Sci., 50: 25-30. https://doi.org/10.1016/j. jdermsci.2007.10.012

Kramer, J., Fahey, M., Santos, R., Carville, A., Wachtman, L. and Mansfield, K., 2010. Alopecia in rhesus macaques correlates with immunophenotypic alterations in dermal inflammatory infilterates consistent with hypersensitivity etiology. J. med. Primatol., 39: 112-122. https://doi.org/10.1111/ j.1600-0684.2010.00402.x

Macias, V.C., Rafael, M., Fernandes, C. and Rosa, J.C., 2013. Diffuse neurofibroma - an uncommon cause of alopecia. Annls Br. Dermatol., 88: 166-169. https://doi.org/10.1590/abd1806-4841.20132170

Novak, M.A. and Meyer, J.S., 2009. Alopecia: Possible causes and treatments, particularly in captive nonhuman primates. Comp. Biol. Med., 59: 18-26.

Panteleyev, A.A., Botchkareva, N.V., Sundberg, J.P., Christiano, A.M. and Ralf, P., 1999. The role of hairless (hr) gene in the regulation of hair follicle catagen transformation. Am. J. Pathol., 155: 159-171. https://doi.org/10.1016/S00029440(10)65110-3 
Pedrosa, A., Nogueira, A., Morais, P., Duarte, A.F., Pardal, J., Mota, A. and Azevedo, F., 2013. Photoletter to the editor: Congenital atrichia associated with an uncommon mutation of $H R$ gene. J. Dermatol. Case Rep., 7: 18-19. https://doi. org/10.3315/jdcr.2013.1125

Potter, G.B., Beaudoin, G.M.J. III., DeRenzo, C.L., Zarach, J.M., Chen, S.H. and Thompson, C.C., 2001. The hairless gene mutated in congenital hairloss disorders encodes a nuclear receptor corepressor. Gene Develop., 15: 2687-2691. https:// doi.org/10.1101/gad.916701

Roy, B., Leszyk, J.D., Mangus, D.A. and Jacobson, A., 2015. Nonsense suppression by near-cognate tRNA employs alternative base pairing at codon position 1 and 3. Proc. natl. Acad. Sci. USA., 112: 30383043. https://doi.org/10.1073/pnas. 1424127112

Sacchidanand, S., Sahana, M.S., Hiremagalore, R. and Asha, G.S., 2012. Congenital atrichia associated with situs inversus and mesocardia. Int. J. Trichol., 4: 181-183. https://doi.org/10.4103/09747753.100093

Sadowski, M.I., and Jones, D.T., 2007. Benchmarking template selection and model quality assessment for high-resolution comparative modeling. Protein, 69: 476-485. https://doi.org/10.1002/prot.21531

Santos, Z., Avci, P. and Hamblin, M.R., 2015. Drug discovery for alopecia: Gone today, hair tomorrow. Exp. Opin. Drug Dis., 3: 269-292. https://doi.org/1 0.1517/17460441.2015.1009892

Taqddus, A., Saad, A.B.A., Pasha, B., Latif, M., Safdar, S., Shaikh, R.S., Ali, M. and Iqbal, F., 2014. Association of endothelial nitric oxide synthase (eNOS) gene polymorphism (Glu 298 Asp) with coronary artery disease in subjects from Multan (Pakistan). Pak. J. pharmceut. Sci., 27: 357-363.

Wali, A., Ansar, M., Khan, M.N. and Ahmad, W., 2006. Atrichia with papular lesions resulting from a novel insertion mutation in the human hairless gene. $J$. clin. exp. Dermatol., 31: 695-698. https://doi. org/10.1111/j.1365-2230.2006.02165.x

Werner, B. and Brenner, F.B., 2012. Clinical and histological challenge in the differential diagnosis of diffuse alopecia: Female androgenetic alopecia, telogen effluvium and alopecia areata Part II. An. Bras. Dermatol., 6: 884-890. https://doi. org/10.1590/S0365-05962012000600010 